\title{
Language learning in Down syndrome: The speech and language profile compared to adolescents with cognitive impairment of unknown origin
}

\author{
Robin S. Chapman, Ph.D. \\ Professor Emerita, Dept. of Communicative Disorders, \& Principal Investigator, Waisman Center \\ University of Wisconsin-Madison
}

\begin{abstract}
Children and adolescents with Down syndrome show an emerging profile of speech and language characteristics that is typical of the syndrome (Chapman \& Hesketh, 2000; Chapman, 2003; Abbeduto \& Chapman, 2005) and different from typically developing children matched for nonverbal mental age, including expressive language deficits relative to comprehension that are most severe for syntax, and, in adolescence, strengths in comprehension vocabulary, improvements in expressive syntax, but losses in comprehension of syntax (Chapman, Hesketh \& Kistler, 2002). Here we compare 20 adolescents with Down syndrome tol 6 individuals with cognitive impairment of unknown origin, statistically matched for age and nonverbal mental age, to show that the age-related strengths in vocabulary comprehension are not limited to the Down syndrome phenotype, but are limited to a certain type of vocabulary test: for both groups, performance on the Peabody Picture Vocabulary Test-3 is significantly greater than performance on the vocabulary subtest of the Test of Auditory Comprehension of Language-3, which does not differ from the syntax comprehension subtests. Vocabulary size, but not conceptual level, is a strength for adolescents with cognitive impairment. In contrast, deficits in auditory-verbal working memory, syntax and vocabulary comprehension, and narration of picture-books without an opportunity to preview them are all specific to the adolescent group with Down syndrome. The expressive language deficit disappears when a preview opportunity and picture support is given.
\end{abstract}

Keywords: Down syndrome, adolescents, language, working memory, phenotype, cognitive impairment of unknown origin

\section{The developmental emergence of speech and language skills}

Studies of children and adolescents with Down syndrome have identified a specific behavioural phenotype marked by deficits in expressive language, especially in speech intelligibility, syntax, and grammatical morphology, accompanied by deficits in phonological working memory and strengths in vocabulary comprehension compared to nonverbal mental age (see Miller, Leddy \& Leavitt, 1999; Chapman \& Hesketh, 2000, Chapman, 1999, 2003, and Abbeduto \& Chapman, 2005, for reviews). The expressive language and working memory deficits are more marked than comprehension skills, nonverbal visual problem solving skills, daily living, or social skills (Dykens, Hodapp \& Evans, 1994).
In adolescence, additional divergence in skill levels has been found, including actual losses in longitudinal development of syntax comprehension, gains in longitudinal development of complex syntax, and superior strength in vocabulary comprehension compared to nonverbal mental age matched controls (Chapman \& Hesketh, 2001, Chapman, Seung, Schwartz \& Kay-Raining Bird, 2000). The rate of development of nonverbal visual short-term memory skills, in six years of longitudinal study, is slower than nonverbal pattern analysis skills and predicts the rate of syntax comprehension growth or loss in adolescence (Chapman, Hesketh \& Kistler, 2002).

Vocabulary comprehension strengths emerge in adolescence, but whether this is unique to the phenotype, or a product of increased life experience, is not clear. Facon, Facon-Bollingier and Gruber (2002) have reported that, for 
a group of 126 children and adolescents with mental retardation, selected without regard to aetiology, chronological age explains a significant (17\%) amount of variance in PPVTrevised performance when entered after a nonverbal mental age measure; but makes no additional contribution in predicting syntax comprehension, as measured by the Test for the Reception of Grammar. Consistent with this view, Facon earlier reported a significant correlation between chronological age and the age-equivalent score on the French version of the PPVT for 29 children aged 6 to 13 years with Down syndrome (Facon, Gruber \& Gardez, 1998) and for children and adolescents with mental retardation (Facon \& Facon-Bollengier, 1997).

In our own work (Miolo, Chapman \& Sindberg, 2005), the strength of adolescents' performance on the PPVT has been replicated in a second study of individuals with Down syndrome (the group included here), where it appears attributable to the nature of the test used. PPVT strengths in performance were not replicated on a vocabulary test more nearly based on lexical selection for conceptual difficulty (the Vocabulary subtest of the Test of Auditory Comprehension of Language-3); in contrast, picturability and frequency of use governed selection of PPVT items. By this argument, other adolescent groups with cognitive impairment of unknown origin should also show strengths on the PPVT-3, but mental-age-commensurate performance on the vocabulary subtest of the TACL-3. We test that prediction here, comparing the phenotype for Down syndrome in adolescence to a group with cognitive disability of unknown origin matched statistically for chronological age and nonverbal mental age, asking if the adolescent pattern of Down syndrome language and cognitive skills more broadly characterises individuals with cognitive impairment, or is specific to Down syndrome; and whether vocabulary performance varies with test.

\section{Method}

\section{Participants}

We compared the language profiles of 20 adolescents with Down syndrome, aged 12 to 21 , with 16 adolescents whose cognitive impairment was of unknown origin and whose visual nonverbal cognition was statistically matched to the Down syndrome group (see Table 1). The groups did not differ significantly in chronological age or raw scores on the Bead Memory and Pattern Analysis subtests of the Stanford Binet, $4^{\text {th }}$ edition, the tests of nonverbal cognition. All participants met the inclusionary criteria of a) monolingual use of spoken English at home and school; b) mean length of utterance, from a narrative language sample, of at least 2.0 morphemes; c) pure-tone average of less than $40 \mathrm{~dB}$ in at least one ear; d) chronological age between 12 and 21 years; and e) no history of severe behaviour, attention, or psychiatric disorder or developmental disorder other than cognitive impairment, according to parental report.

\section{Testing}

In a three-hour protocol we administered a hearing screening (carried out by an audiologist) at $20 \mathrm{~dB}$ for 500,1000 , and $2000 \mathrm{~Hz}$, and further testing to establish a pure tone average less than $40 \mathrm{~dB}$ for at least one ear if these were failed. Hearing status was scored 1 if the individual passed all $20 \mathrm{~dB}$ screening frequencies and 0 if they failed any. The protocol also included tests of nonverbal cognition (the Bead Memory and Pattern Analysis subtests of the Stanford Binet- $4^{\text {th }}$ ed., Thorndike, Hagen \& Sattler, 1986), vocabulary comprehension (Peabody Picture Vocabulary Test-3, Dunn \& Dunn, 1997), and the vocabulary subtest of the Test of Auditory Comprehension of Language-3 (CarrowWoolfolk, 1999), syntax comprehension (grammatical 
morpheme and elaborated phrase subtests of the TACL-3), interview language samples with the examiner on topics of school, favourite activities, friends, and family; and narrative language samples derived from telling stories to two different wordless picturebooks, one of which they had the counterbalanced opportunity to preview. We also administered tests of auditory verbal working memory, including the Kaufman-ABC number recall task (digit span; Kaufman \& Kaufman, 1983) and the Nonword Repetition Test (Dollaghan \& Campbell, 1998); see Miolo, Chapman and Sindberg, 2005, for a more detailed account of these procedures.

\section{Transcription}

Interview and narrative language samples were transcribed from audiotape using SALT conventions for spelling and morpheme marking (Miller \& Chapman, 1996) with the opportunity to listen at least three times to the utterance; transcribers also had the picture books available. Transcriptions were reviewed for reliability of morpheme transcription and utterance segmentation and retranscribed if reliability did not reach $90 \%$ for each. The SALT program was used to compute measures of mean length of utterance in morphemes (MLU) for the samples.

\section{Results}

\section{Cognition}

The group with cognitive impairment of unknown origin (CI) was selected to be statistically similar to the group with Down syndrome in chronological age and the mean of the nonverbal visual Stanford Binet subtests and did not differ significantly in those measures. Nor did the groups differ significantly on the difference between their pattern analysis and bead memory subtests; performance on pattern analysis was slightly better, by .42 (1.07) and .28 (3.25) years, for the Down syndrome and cognitive impairment groups respectively, though the variability was significantly greater in the CI group.

\section{Working memory}

With respect to phonological working memory measures, the groups did not differ significantly on two measures, the total phonemes correct out of 96 on the Nonword Repetition Test or the longest string recalled on the K-ABC digit span test. They did, however, differ significantly $(p<.05 \mathrm{l}$ tailed) on performance on 4 -syllable words of the Nonword Repetition Test (Down syndrome $\mathrm{X}=13.50, \mathrm{SD}=6.31$; cognitive impairment of unknown origin $=18.00, \mathrm{SD}=9.49$ ) and on the age-equivalent and age-based standard scores for the K-ABC Number Recall digit span test, with a significantly $(p<.05,1$-tailed) poorer performance in the group with Down syndrome (for standard scores, $\mathrm{X}=63.00$, $\mathrm{SD}=10.31$ ), vs. the group with cognitive impairment of unknown origin (for standard scores, $\mathrm{X}=73.67, \mathrm{SD}=14.94$ ); age-equivalent scores are reported in Table 1 . Thus the auditory-verbal working memory deficit that is found in Down syndrome appears to be part of its specific phenotype, rather than a general feature of cognitive impairment.

\section{Language comprehension and production}

Multivariate tests, followed by univariate ones, evaluated group effects for comprehension (raw scores on the PPVT3 , and the TACL-3 subtests for vocabulary, grammatical morphemes, and elaborated phrases) and for production (mean length of utterance measures in morphemes for complete and intelligible utterances in 9 minutes of interview and the two narrative samples from wordless picture books, previewed or not, excluding yes-no answers and pragmatic acknowledgments in the interview samples, and irrelevant utterances in the narrative).

All comprehension measures revealed significantly poorer performance in the group with Down syndrome compared to the group with cognitive impairment of unknown origin (multivariate test of group $F(4,30)=2.51, p<.051$-tailed, partial eta-squared $=.25$ ) Univariate tests are significant, $p<.05$, for TACL-vocabulary raw score $(F(1,33)=5.90$, partial eta-squared $=.152)$; for TACL-grammatical morphemes raw score $(F(1,33)=10.03$, partial eta-squared $=.23$; for TACL-elaborated phrases raw score $(F(1,33)=9.09$, partial eta-squared $=.22$; and for PPVT raw score $(F(1,33)=6.46$, partial-eta squared $=.16)$. Thus the loss of comprehension skills in adolescence appears specific to Down syndrome, and is poorer for both syntax comprehension and vocabulary comprehension than the group with cognitive impairment of unknown origin.

Though production measures were lower in the group with Down syndrome, the overall multivariate comparison was not significant, $F(3,30)=1.68, p=.10$ l-tailed. The univariate tests revealed that the non-previewed narrative was significantly shorter in mean utterance length in the Down syndrome group, univariate $F(1,32)=2.98, p<.05$ l-tailed, with a partial eta-squared of .09. The means (standard deviations) for DS were 6.19 (2.39) and for CI were 7.85 (3.23), $\mathrm{n}=14$ for the latter group. The effect of not having an opportunity to preview the picture book is more limiting on MLU for adolescents with Down syndrome than for adolescents with cognitive impairment of unknown origin, but with that opportunity, and with picture support for a narrative sample that reveals more complex syntax, the expressive language deficit evident in childhood years is not found here, in comparison to a group of adolescents whose cognitive impairment is of unknown origin. These effects of language sampling procedures on measured MLU are discussed further for the adolescents with Down syndrome by Miles, Chapman and Sindberg (2006).

\section{Are vocabulary strengths greater on the PPVT than the TACL?}

Within group paired t-tests evaluated the difference between PPVT-3 and TACL-3 Vocabulary age-equivalent scores (see Table 1); these scores were significantly $(p<.01)$ higher for the PPVT-3 in each group, confirming that in both groups, vocabulary as sampled by the PPVT is greater 
than expectations based on the TACL vocabulary test, or on mental age or syntax comprehension performance. (Comparisons within groups of TACL-3 subscores vocabulary vs. morpheme comprehension or elaborated phrases were not significant). When we examined the typically developing syntax-comprehension control group $(n=20)$ reported elsewhere (Miolo, Chapman \& Sindberg, 2005), that group showed no significant difference in TACL-3 and PPVT3 age-equivalent scores, with means (SDs) of 5.32 (1.61) and 5.18 (1.61) respectively - as, indeed, one would expect with adequate norming of the tests. Thus, the significantly better performance of the Down syndrome and cognitively impaired groups on the PPVT-3, relative to syntax comprehension, is real, and appears attributable to chronological age and the additional life experience it affords.

\section{The role of hearing}

A higher proportion of the group with Down syndrome failed at least one of the hearing screening frequencies at $20 \mathrm{~dB}(50 \%$, vs. $20 \%)$. To assess whether profile differences in comprehension and production were attributable to hearing, group status, or both, we carried out hierarchical regression analysis on the full group, adding hearing as the first level variable and group membership (Down syndrome or intellectual impairment of unknown origin) as the second, for the comprehension measures and the MLU measures.

\section{Production}

Hierarchical linear regression of hearing status, followed by group membership, for the MLU variables revealed that hearing status only accounted for significant $(p<.05)$ variation in the MLU interview measure, Fchange $(1,31)=5.006$, $p<.05, \mathrm{R}$-square $=.14$. There was no additional effect of group. (Neither hearing nor group accounted for significant variance in the MLU-average narrative measure).

\section{Comprehension}

For PPVT-3 raw score, hearing did not explain significant variance (Fchange $(1,33)=1.188, p>.05)$; group, entered second, did (Fchange $(1,32)=5.509, p<.05$, R-square change $=.142$. For TACL-vocabulary raw score, hearing explained significant variance $($ Fchange $(1,33)=4.380$, $p<.05)$, R-square $=.12$, and group explained additional significant variance $(\operatorname{Fchange}(1,32)=4.117, p<.05)$, Rsquare change $=.10$. Thus the TACL vocabulary subtest is more affected by hearing status than the PPVT. For TACLgrammatical morphemes raw score, hearing explained significant variance $($ Fchange $(1,33)=7.090, p<.05)$, R-square $=.18$, followed by group membership (Fchange $(1,32)=$ $6.217, p<.05)$, R-square change $=.14$. For TACL-elaborated phrase raw score, hearing did not explain significant variance $($ Fchange $(1,33)=2.734, p>.05)$. Group membership, entered second, did explain significant variance $($ Fchange $(1,32)=7.422), p<.05, \mathrm{R}$-square change $=.17)$.

In summary, hearing status accounted for differences in MLU-interview. Group membership accounted for dif- ferences in comprehension performance on vocabulary tests, with hearing additionally contributing to the TACLvocabulary test but not the PPVT. Hearing and group membership accounted for the differences in grammatical morpheme comprehension; group alone, for the poorer performance on elaborated phrase comprehension by the adolescents with Down syndrome. Thus, the poorer comprehension performance in the group with Down syndrome is attributable to the phenotype, with hearing status (also arising from DS) contributing to TACL-vocabulary and grammatical morpheme comprehension but not PPVTvocabulary, nor TACL-elaborated phrase comprehension.

\section{Predicting individual differences within groups}

We further analysed predictors of individual difference in comprehension and production measures within each group separately.

\section{Down syndrome}

Nonverbal cognition and visual working memory were not significantly correlated with any of the comprehension or production measures, and were excluded from consideration. The correlations of phonological working memory measures (K-ABC digit span, Non-word Repetition Test) and hearing status with the language measures are shown in Table 2. Comprehension measures were significantly related to the NRT, for TACL-vocabulary and elaborated phrases; and to hearing, for TACL-grammatical morphemes. MLU in both narrative and interview samples was significantly predicted by the combination of phonological working memory measures in regression analyses, with approximately equal contributions by each measure. For MLU narrative, R-squared=.53, $F(2,17)=9.50, p<.002$; for MLU interview, R-squared $=.51, F(2,17)=8.99, p<.002$.

Table 2. Down syndrome $(n=20)$ : Correlations between predictor variables and comprehension and production measures

\begin{tabular}{lccc}
\hline Measures & \multicolumn{2}{c}{ Working Memory: } & Hearing \\
& K-ABC & NRT & \\
\hline TACL-vocab & .39 & $.49 *$ & .37 \\
TACL-gram morphemes & .39 & .21 & $.51^{*}$ \\
TACL-elab phrases & .25 & $.58^{* *}$ & .30 \\
PPVT & .32 & .28 & .18 \\
MLU Narrative & $.57^{* *}$ & $.58^{* *}$ & .18 \\
MLU Interview & $.59^{*} *$ & $.54^{*}$ & .33 \\
\hline
\end{tabular}

*p $<.05 * * p<.01$

\section{Cognitive impairment of unknown origin}

Nonverbal cognition (SB Pattern Analysis) and working memory (SB Bead memory; K-ABC digit span, Non-word repetition test) measures were examined as predictors of individual difference in comprehension and production measures within the group with cognitive impairment 
of unknown origin (see Table 3). In this relatively small$\mathrm{n}$ group, only K-ABC digit span contributed significant variance in models predicting comprehension and narrative production performance; addition of other, moderately correlated, predictors did not add significant additional explained variance.

Table 3. Cognitive impairment of unknown origin $(n=16)$ : Correlations between predictor variables and comprehension and production measures

\begin{tabular}{|c|c|c|c|c|}
\hline \multirow[t]{2}{*}{ Measures } & \multicolumn{2}{|c|}{ Working Memory } & \multicolumn{2}{|c|}{ Nonverbal Cognition } \\
\hline & K-ABC & NRT & Bead & Pattern \\
\hline TACL-vocab & $.50 * \mathrm{a}$ & .23 & $.50 * \mathrm{a}$ & $.30^{\mathrm{a}}$ \\
\hline $\begin{array}{l}\text { TACL-gram } \\
\text { morphemes }\end{array}$ & $.78 * *$ b & $.5 I^{*} \mathrm{a}$ & $.54 *$ & $.51^{\mathrm{a}}$ \\
\hline $\begin{array}{l}\text { TACL-elab } \\
\text { phrases }\end{array}$ & $.73^{* * * a}$ & .47 & .49 & $.35^{\mathrm{a}}$ \\
\hline PPVT & $.65^{* * *}$ & .35 & .28 & $.51 *$ \\
\hline MLU Narrative & $.60 * \mathrm{c}$ & $.57^{*} \mathrm{~b}$ & .41 & $.45^{b}$ \\
\hline MLU Interview & $.52^{c}$ & $.42^{\mathrm{b}}$ & $.32^{\mathrm{b}}$ & $.4 \mathrm{I}^{\mathrm{b}}$ \\
\hline
\end{tabular}

\section{Summary of predictors of individual variation}

These foregoing analyses suggest that phonological working memory plays an important role in comprehension and production performance by both groups; but that the Nonword Repetition Test, with its greater component of longterm knowledge of phonotactics, is more important for the Down syndrome group than the index of phonological store and rehearsal provided by the K-ABC digit span in the CI group. Additionally, hearing status affects DS grammatical morpheme comprehension and interview-MLU.

\section{Phenotypic profiles}

The significant differences between the groups (matched in nonverbal visual cognition) in auditory-verbal working memory, comprehension measures, and the non-previewed narrative language sample confirm a specific behavioural phenotype for language and cognition in adolescents with Down syndrome, compared to individuals for whom the origin of cognitive impairment is unknown. Compared with the adolescents with cognitive impairment of unknown origin, the adolescents with Down syndrome show poorer auditory-verbal working memory skills, poorer comprehension skills, and more limited narrative language skill when no opportunity to preview the story is provided. Both groups showed strengths, relative to their nonverbal mental age performance, on vocabulary comprehension measured by the PPVT (reflecting vocabulary size), but not on vocabulary comprehension on the TACL-vocabulary subtest (reflecting, to a greater degree, conceptual level of vocabulary).
The profile for adolescents with Down syndrome differs from that reported for children with Down syndrome in its age-associated strengths in vocabulary size (PPVT), deficits relative to non-verbal age in syntax comprehension, and improved performance on narratives produced with previewing and picture support.

\section{Limitations of study}

The group with cognitive impairment of unknown origin was smaller than we had hoped; it proved difficult to find individuals for whom attention deficit disorder or other conditions (seizures, psychiatric diagnoses) were not reported, and the origins of cognitive impairment have been identified in many more individuals today than in the past. Indeed, the comparison group is a moving target, as research sorts out genetic and environmental causes. Thus the predictors for the CI group variation should be especially viewed with caution.

\section{Acknowledgments}

This research was supported by National Institutes of Health grant R01-HD-23353 to R. Chapman, with additional support from the National Down Syndrome Society. Thanks to the parents and participants who made the work possible, and to the many colleagues who participated in this research, including Cynthia Bridge, Katharine Gigstead, Dr. Linda J. Hesketh, Dr. Sally Miles, Dr. Giuliana Miolo, and Heidi Sindberg.

\section{Correspondence}

Robin Chapman • Waisman Center, 1500 Highland Ave., Madison, WI 53705 USA • chapman@waisman.wisc.edu

\section{References}

Abbeduto, L. \& Chapman, R.S. (2005). Language and communication skills in children with Down syndrome and Fragile x. In P. Fletcher \& J. Miller, (Eds.), Trends in language acquisition research, vol 4: Developmental theory and language disorders. Amsterdam, NL: John Benjamins.

Carrow-Woolfolk, E. (1999). Test for Auditory Comprehension of Language-Third Edition. Circle Pines, MN: AGS.

Chapman, R.S. (1999). Language and cognitive development in children and adolescents with Down syndrome. In J.F. Miller, L.A. Leavitt, and M. Leddy, (Eds.), Improving the Communication of People with Down Syndrome. (pp. 41-60). Baltimore, MD: Paul H. Brookes Publishing.

Chapman, R.S. (2003). Language and communication in individuals with Down syndrome. (pp. 1-34) In L. Abbeduto (Ed.), International Review of Research in Mental Retardation: Language and Communication, vol. 27. Academic Press.

Chapman, R.S. \& Hesketh, L.J. (2000). Behavioural phenotype of individuals with Down syndrome. Mental Retardation and Developmental Disability Research Reviews, 6, 84-95.

Chapman, R.S. \& Hesketh, L.J. (2001). Language, cognition, and short-term memory in individuals with Down syndrome. Down Syndrome Research and Practice, 7, 1-7. 
Chapman, R.S., Hesketh, L.J. \& Kistler, D. (2002). Predicting longitudinal change in language production and comprehension in individuals with Down syndrome: Hierarchical linear modeling. Journal of Speech, Language, and Hearing Research, 45, 902-915.

Chapman, R.S., Seung, H-K., Schwartz, S.E. \& Kay-Raining Bird, E. (2000). Predicting language development in children and adolescents with Down syndrome: The role of comprehension. Journal of Speech, Language, and Hearing Research, 43, 340-350.

Dollaghan, C. \& Campbell, T. (1998). Nonword repetition and child language impairment. Journal of Speech, Language, and Hearing Research, 41, 1136-1146.

Dunn, L.M. \& Dunn, L.M. (1997). The Peabody Picture Vocabulary Test-Third Edition. Circles Pines, MN: AGS:

Dykens, E.M., Hodapp, R.M. \& Evans, D.W. (1994). Profiles and development of adaptive behaviour in children with Down syndrome. American Journal on Mental Retardation, 98, 580-587.

Facon, B. \& Facon-Bollengier, T. (1997). Chronological age and Peabody Picture Vocabulary Test performance of persons with mental retardation: New data. Psychological Reports, 81, 1232-1234.

Facon, B., Facon-Bollengier, T. \& Grubar, J-C. (2002). Chronological age, receptive vocabulary, and syntax comprehension in children and adolescents with mental retardation. American Journal on Mental Retardation, 107, 91-98.

Facon, B., Grubar, J.C. \& Gardez, C. (1998). Chronological age and receptive vocabulary of persons with Down syndrome. Psychological Reports, 82, 723-726.

Kaufman, K. \& Kaufman, N. (1983). Kaufman Assessment Battery for Children. Circle Pines, MN: AGS.

Miles, S., Chapman, R. \& Sindberg, H. (2006). Sampling context affects MLU in the language of adolescents with Down syndrome. Journal of Speech, Language, and Hearing Research, 49, 325-337.

Miller, J.F. \& Chapman, R.S. (1996). Systematic Analysis of Language Transcripts (Computer Software), Language Analysis Laboratory, University of Wisconsin-Madison.

Miller, J.F., Leddy, M.G. \& Leavitt, L.A. (1999) Improving the Communication of People with Down Syndrome. Baltimore, MD: Paul H. Brookes Publishing.

Miolo, G., Chapman, R.S. \& Sindberg, H. (2005). Sentence comprehension in adolescents with Down syndrome and typically developing children: Role of sentence voice, visual context, and auditory-verbal short-term memory. Journal of Speech, Language, and Hearing Research, 48, 172-188.

Thorndike, R.L., Hagen, E.P. \& Sattler, J.M. (1986). Stanford-Binet Intelligence Scale ( $4^{\text {th }} \mathrm{ed}$.). Chicago: Riverside. 